\title{
Compositional Stability of Approximately Symmetric Systems: Initial Results
}

\author{
Bill Goodwine
}

\begin{abstract}
This paper considers nonlinear control systems that are approximately symmetric, and extends some prior work of the author related to stability of symmetric systems to the case where the system is not exactly symmetric. Many engineering systems are composed of components that are nominally identical, but due inherent variability in physical systems, can not be exactly symmetric. By exploiting the baseline symmetric structure of the system and constraining the deviations from exact symmetry, stability results are derived that are independent of the number of components in the system. This paper specifically focuses on the application of LaSalle's Invariance Principle to approximately symmetric systems, which has broad applicability. The main utility of the stability result is one of scalability or compositionality because the main result shows that if the system is stable for a given number of components, under appropriate conditions, stability is then guaranteed for a larger system composed of the same type of components which are interconnected in a manner consistent with the smaller system.
\end{abstract}

Index Terms - symmetric systems, multiagent coordination, nonlinear systems, compositionality

\section{INTRODUCTION}

Many research efforts consider the analysis of composability and compositionality of control systems, especially cyber-physical systems [1], [2]. In this paper conditions are determined under which a stable approximately symmetric system remains stable if additional components are added in a structured manner. This work is an extension of the work presented in [3], and is also related to some of our prior work in [4]-[9]. The main application focus in this paper is formation control of mobile robotic systems. Related work for formation control includes [10]-[17] and many others. Symmetries have been considered in prior work in control systems, such as in [18]-[20], but the idea has not yet been fully exploited for mainstream results.

The main idea of the result in this paper is that if a system is characterized by a symmetry, then there is quite a bit if structure present in the equations of motion that may be exploited for control and analysis purposes. This was the primary theme in our prior results in [3]. The results in that paper formalized the definition of a symmetric system, and based on that definition defined an equivalence relation among symmetric systems with a different number of components. Then it determined conditions under which stability is a property that is invariant across the equivalence class of

Bill Goodwine is with the Department of Aerospace \& Mechanical Engineering at the University of Notre Dame, Notre Dame, IN 46556 USA. Email: billcontrols.ame.nd.edu.

The partial support of the National Science Foundation under the CPS Large Grant No. CNS-1035655 is gratefully acknowledged. systems defined by the equivalence relation. This allows a control engineer to analyze only one system (presumably the smallest one), and be able to infer stability for all larger equivalent systems that contain more components. This paper extends those results to allow the exact symmetry to be broken. It is emphasized that the symmetry breaking does not necessarily have to be small.

The rest of this paper is organized as follows. Section II outlines the definition of a symmetric system, equivalence relations among different symmetric systems and equivalence classes of symmetric systems. Section III presents the nonlinear stability results for approximately symmetric systems. Section IV presents an example of the application of these results. Finally, Section V outline conclusions and future work.

\section{SyMmetric SYSTEMS}

This section gives an overview of symmetric systems and the relationship among symmetric systems with different numbers of components. As a motivational example, consider a formation of large number of identical mobile robots where each robot has a control law that attempts to control it so that it maintains a desired distance from its neighbors. Intuitively if more of the same type of robots with the same control law are added to the formation, or conversely if some are removed, the properties of the formation as a whole should normally not drastically change.

The "basic building block" (extended to the nonlinear case from [21]) in one spatial dimension is illustrated in Figure 1. The outputs from the component are $w^{-}(t)$ and $w^{+}(t)$, and the inputs are $u, v^{-}(t)$ and $v^{+}(t)$. The signals $v^{ \pm}$will represent the effects of the coupling with the other components and $u$ are the control inputs. We consider systems of the form

$$
\begin{aligned}
\dot{x}_{i}(t) & =f_{i}\left(x_{i}(t)\right)+\sum_{j=1}^{m_{i}} g_{i, j}\left(x_{i}(t)\right) u_{i, j}(t) \\
w_{i}^{-}(t) & =w_{i}^{-}\left(x_{i}(t)\right) \\
w_{i}^{+}(t) & =w_{i}^{+}\left(x_{i}(t)\right)
\end{aligned}
$$

where the control law may be a function of the outputs from the neighbors so the control input for component $i$ Equation 1 can be written as

$$
u_{i, j}(t)=u_{i, j}\left(x_{i}(t), w_{i-1}^{+}\left(x_{i-1}(t)\right), w_{i+1}^{-}\left(x_{i+1}(t)\right)\right) .
$$

The equations and building block so far have allowed for interconnections only in one dimension. Of course, systems may be spatially interconnected in dimensions greater than 


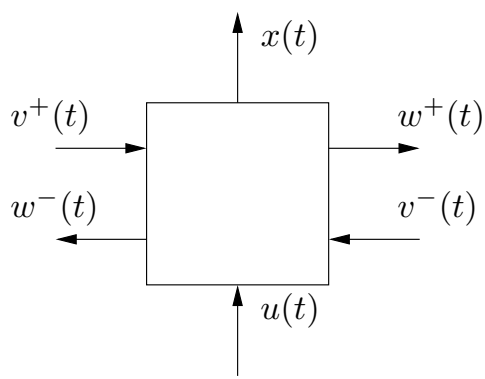

Fig. 1. System building block in one spatial dimension.



Fig. 2. System topology for Example 2.

one or with a different type of periodicity, as is illustrated in Figure 2, which illustrates that interconnections are not necessarily limited to connections with only two neighbors in each dimension. To generalize this, the types of systems considered in this paper will have components that are members of groups. Recall, a group is a set, $G$ with

1) a binary associative operation, $\sigma: G \times G \rightarrow G$,

2) an identity element $e$ such that $\sigma(e, g)=\sigma(g, e)=g$ for all $g \in G$, and

3) for every $g \in G$ there exists an element $g^{-1} \in G$ such that $\sigma\left(g, g^{-1}\right)=\sigma\left(g^{-1}, g\right)=e$.

Let $|G|$ denote the number of elements in a set $G$.

If $X$ is a subset of a group $G$, then the smallest subgroup of $G$ containing $X$ is called the subgroup generated by $X$. For simplicity, for the rest of this paper we will assume that if $s \in X$, then $s^{-1} \in X$ as well. We will use a "multiplication" notation instead of $\sigma$ for the operation, i.e., $g_{1} g_{2}=\sigma\left(g_{1}, g_{2}\right)$. Constraints among the generators are given by relations of the form $s_{1} s_{2} \ldots s_{m}=e$ for $s_{1}, \ldots, s_{m} \in X$. Finally, we represent systems by a Cayley graph, which is a directed graph with vertices that are the elements of a group, $G$, generated by the subset $X$, with a directed edge from $g_{1}$ to $g_{2}$ only if $g_{2}=s g_{1}$ for some $s \in X$ (see [22] for a more extensive exposition). In our systems, a edge from node $g_{1}$ to $g_{2}$ represents that a coupling input to $g_{2}$ is equal to an output from $g_{1}$.

Example 1: Consider the ring of components illustrated in Figure 2. Each vertex has edges connecting to four other vertices and hence the system is generated by four elements. Let $g$ denote a vertex, i.e., $g \in\{-2,-1,0,1, \ldots, N-3\}=$ $G$. Consider the subset of generators $X=\{-2,-1,1,2\}$, the group operation to be addition between integers and the relation $s^{N}=e=0$. This relation makes the group operation of addition to be $\bmod N$, and hence the group is the quotient of the set of integers $\mathbb{Z}$ where elements of $\mathbb{Z}$ that differ by an integer multiple of $N$ are equivalent. The Cayley graph is illustrated in Figure 2.

For a system on the group $G$ with the set of generators $X=\left\{s_{1}, s_{2}, \ldots, s_{|X|}\right\}$, the state variable corresponding to $g \in G$ is denoted by $x_{g}$, the set of neighbors of component $g \in G$ is denoted by $X g=\left\{s_{1} g, s_{2} g, \ldots, s_{|X|} g\right\}$, the states of the neighbors by $x_{X g}$ and the states of the neighbors of the neighbors by $x_{X X g}$. For a component $g$, the set of outputs is denoted by $\left\{w_{g}^{s_{1}}, w_{g}^{s_{2}}, \ldots, w_{g}^{s_{|X|}}\right\}$ and similarly the set of inputs $\left\{v_{g}^{s_{1}}, v_{g}^{s_{2}}, \ldots, v_{g}^{s_{|X|}}\right\}$. Subsequently the definition of a periodically interconnected system will require that $w_{g}^{s}$, the output from $g$, is taken as an input to component $s g$. The dynamics of a component, $g \in G$ are represented by

$$
\begin{aligned}
\dot{x}_{g}(t)= & f_{g}\left(x_{g}(t)\right) \\
& +\sum_{j=1}^{m_{g}} g_{g, j}\left(x_{g}(t)\right) u_{g, j}\left(x_{g}(t), v_{g}^{s_{1}}(t), \ldots, v_{g}^{s_{|X|}}(t)\right)
\end{aligned}
$$$$
w_{g}^{s}(t)=w_{g}^{s}\left(x_{g}(t)\right),
$$

for all $s \in X$.

Definition 1: Let $G$ be a group with a set of generators, $X$. A system with components $g \in \mathcal{I} \subset G$ with dynamics given by Equation 3 has periodic interconnections on $\mathcal{I}$ if

$$
v_{g}^{s}(t)=w_{s^{-1} g}^{s}\left(x_{s^{-1} g}(t)\right),
$$

for all $g \in \mathcal{I}$ and $s \in X$. Furthermore, if

$$
\begin{aligned}
f_{g_{1}}(x) & =f_{g_{2}}(x), & g_{g_{1}, j}(x) & =g_{g_{2}, j}(x), \\
w_{g_{1}}^{s}(x) & =w_{g_{2}}^{s}(x), & m_{g_{1}} & =m_{g_{2}}=m
\end{aligned}
$$

for all $s \in X, g_{1}, g_{2} \in \mathcal{I}, x \in \mathbb{R}^{n}$ and $j \in\{1, \ldots, m\}$, then $\mathcal{I}$ forms an orbit of symmetric components. Finally, if the control laws also satisfy

$$
\begin{aligned}
u_{g_{1}, j} & \left(x_{1}, w_{s_{1}^{-1} g_{1}}^{s_{1}}\left(x_{2}\right), \ldots, w_{s_{|X|}^{-1} g_{1}}^{s_{|X|}}\left(x_{|X|+1}\right)\right)= \\
& u_{g_{2}, j}\left(x_{1}, w_{s_{1}^{-1} g_{2}}^{s_{1}}\left(x_{2}\right), \ldots, w_{s_{|X|}^{-1} g_{2}}^{s_{|X|}}\left(x_{|X|+1}\right)\right)
\end{aligned}
$$

for all $g_{1}, g_{2} \in \mathcal{I}, j \in\{1, \ldots, m\}, s \in X$ and $\left(x_{1}, x_{2}, \ldots, x_{|X|+1}\right) \in \mathbb{R}^{n} \times \mathbb{R}^{n} \times \cdots \times \mathbb{R}^{n}$ then the elements of $\mathcal{I}$ form a symmetry orbit. Such a system with a symmetry orbit is called a symmetric system on $\mathcal{I}$. If $\mathcal{I}=G$ it is a symmetric system on $G$. 
Example 2: The main example in this paper, which is a variation from [16], is a system of $N+1$ planar agents with equations of motion for the $i$ th robot given by

$$
\frac{d}{d t}\left[\begin{array}{c}
x_{i} \\
\dot{x}_{i} \\
y_{i} \\
\dot{y}_{i}
\end{array}\right]=\left[\begin{array}{c}
\dot{x}_{i} \\
0 \\
\dot{y}_{i} \\
0
\end{array}\right]+\left[\begin{array}{c}
0 \\
1 \\
0 \\
0
\end{array}\right] u_{i, 1}+\left[\begin{array}{l}
0 \\
0 \\
0 \\
1
\end{array}\right] u_{i, 2}
$$

The goal formation is a regular $(N+1)$-polygon centered at the origin, hence the desired formation distance between components $i$ and $j$ is

$$
d_{i j}= \begin{cases}1, & |i-j|=1 \\ \frac{\sin \left(\frac{2 \pi}{N+1}\right)}{\sin \left(\frac{\pi}{N+1}\right)}, & |i-j|=2\end{cases}
$$

$(\bmod (N+1))$ and the desired distance of robot $i$ to the origin is

$$
r_{i}=\frac{1}{2 \sin \frac{\pi}{N+1}} .
$$

Note that there are an infinite number of configurations which satisfy the conditions for "the desired formation" because "the" formation may be rotated about the origin. Take the control law to be

$$
\begin{aligned}
{\left[\begin{array}{l}
u_{i, 1} \\
u_{i, 2}
\end{array}\right]=} & -\sum_{j}\left[\begin{array}{l}
\frac{\left(\sqrt{\left(x_{i}-x_{j}\right)^{2}+\left(y_{i}-y_{j}\right)^{2}}-d_{i j}\right)}{\sqrt{\left(x_{i}-x_{j}\right)^{2}+\left(y_{i}-y_{j}\right)^{2}}}\left(x_{i}-x_{j}\right) \\
\frac{\left(\sqrt{\left(x_{i}-x_{j}\right)^{2}+\left(y_{i}-y_{j}\right)^{2}}-d_{i j}\right)}{\sqrt{\left(x_{i}-x_{j}\right)^{2}+\left(y_{i}-y_{j}\right)^{2}}}\left(y_{i}-y_{j}\right)
\end{array}\right] \\
& -k_{d}\left[\begin{array}{l}
\dot{x}_{i} \\
\dot{y}_{i}
\end{array}\right]-\left[\begin{array}{l}
k_{o} \frac{\sqrt{x_{i}^{2}+y_{i}^{2}}-r_{i}}{\sqrt{x_{i}^{2}+y_{i}^{2}}} x_{i} \\
k_{o} \frac{\sqrt{x_{i}^{2}+y_{i}^{2}}-r_{i}}{\sqrt{x_{i}^{2}+y_{i}^{2}}} y_{i}
\end{array}\right]
\end{aligned}
$$

where $k_{d}$ and $k_{o}$ are positive constant gains and $j \in$ $\{i-2, i-1, i+1, i+2\}$.

To show that this system has a symmetry orbit where the orbit contains all the robots in the system, first, observe that this system can be represented by the graph illustrated in Figure 2 with $G=\{-2,-1,0,1,2, \ldots, N-3\}$, the group operation to be addition and let $X=\{-2,-1,1,2\}$ with the relation $s^{N}=0, N \geq 5$. Then, the Cayley graph for the system is as illustrated in Figure 2. Also, observe from the control law in Equation 8, the control for robot $i$ depends on its own state as well as the states for robots $i-2, i-1$, $i+1$ and $i+2$, which are equivalent to the four generators. Hence, define each of the outputs for robot $i$ to be the vector of the robot's position, i.e., $w_{i}^{s}=\left[\begin{array}{ll}x_{i} & y_{i}\end{array}\right]^{T}$ where $s \in X=\{-2,-1,1,2$,$\} . Define the inputs to component$ $i \in\{-2,-1, \ldots, N-3\}$ to be $v_{i}^{s}=\left[\begin{array}{ll}x_{i-s} & y_{i-s}\end{array}\right]^{T}, s \in$ $\{-2,-1,1,2\}$ which satisfies Equation 4. The dynamics, as given in Equation 7 satisfy Equation 5. Finally, the feedback law given in Equation 8 satisfies Equation 6. Because these hold for all $i \in\{-2,-1,0, \ldots, N-3\}$ the system has an orbit of symmetric components which contains all the components in the system.

Now, we will define two systems to be equivalent if they have symmetry orbits with identical components which are interconnected in the same manner, but they possibly have a different number of components in the symmetry orbit.

Definition 2: Two symmetric systems on the finite groups $G_{1}$ and $G_{2}$ are equivalent if $G_{1}$ and $G_{2}$ are generated by the same set of generators, $X$,

$$
\begin{aligned}
f_{g_{1}}(x) & =f_{g_{2}}(x), \quad g_{g_{1}, j}(x)=g_{g_{2}, j}(x), \\
w_{s^{-1} g_{1}}^{s}(x) & =w_{s^{-1} g_{2}}^{s}(x)
\end{aligned}
$$

and

$$
\begin{gathered}
u_{g_{1}, j}\left(x_{1}(t), w_{s_{1}^{-1} g_{1}}^{s_{1}}\left(x_{2}(t)\right), \ldots, w_{s_{|X|}^{-1} g_{1}}^{s_{|X|}}\left(x_{|X|+1}(t)\right)\right)= \\
u_{g_{2}, j}\left(x_{1}(t), w_{s_{1}^{-1} g_{2}}^{s_{1}}\left(x_{2}(t)\right), \ldots, w_{s_{|X|}^{-1} g_{2}}^{s_{|X|}}\left(x_{|X|+1}(t)\right)\right)
\end{gathered}
$$

for all $g_{1} \in G_{1}, g_{2} \in G_{2}, s \in X, x \in \mathbb{R}^{n}$, $\left(x_{1}, x_{2}, \ldots, x_{|X|+1}\right) \in \mathbb{R}^{n} \times \mathbb{R}^{n} \times \cdots \times \mathbb{R}^{n}$ and $j \in$ $\{1, \ldots, m\}$ where $m=m_{g_{1}}=m_{g_{2}}$.

Example 3: Continue Example 2 and consider two systems with components that satisfy Equation 7 and components belonging to

$$
\begin{aligned}
& G_{1}=\{-2,-1,0,1,2, \ldots, N-3\} \\
& G_{2}=\{-2,-1,0,1,2, \ldots, M-3\}
\end{aligned}
$$

where $M>N$. Because the dynamics of all the components are identical and the feedback definitions are identical, these systems are equivalent. Both have generating sets $X=$ $\{-2,-1,1,2\}$ with the only difference being the relation for $G_{1}$ is $s^{N}=0$ and the relation for $G_{2}$ is $s^{M}=0$. $\diamond$

For notational convenience, we will concatenate all the states and vector fields from each component into one system description of the form, $\dot{x}_{G}=f_{G}\left(x_{G}\right)+g_{G}\left(x_{G}\right) u(t)$ where

$$
f_{G}\left(x_{G}\right)=\left[\begin{array}{c}
f_{g_{1}}\left(x_{g_{1}}\right) \\
f_{g_{2}}\left(x_{g_{2}}\right) \\
\vdots \\
f_{g_{|G|}}\left(x_{g_{|G|}}\right)
\end{array}\right], \quad g_{G}\left(x_{G}\right)=\left[\begin{array}{c}
g_{g_{1}}\left(x_{g_{1}}\right) \\
g_{g_{2}}\left(x_{g_{2}}\right) \\
\vdots \\
g_{g_{|G|}}\left(x_{g_{|G|}}\right)
\end{array}\right]
$$

and $x_{G}=\left[\begin{array}{lll}x_{g_{1}} & \cdots & x_{g_{|G|}}\end{array}\right]^{T}$.

\section{Stability of ApProximately Symmetric SYSTEMS}

This section presents the compositionality stability results for approximately symmetric systems. The results allow us to infer stability of a whole equivalence class of systems based on the stability of one of the members of the class. We first the previous result upon which this work is based. Proposition 1 concerns negative (semi)definiteness of the derivative of a Lyapunov function for each member of an equivalence class of symmetric systems. Then Proposition 3 extends this result to approximately symmetric systems.

Proposition 1: Given a symmetric system on a finite group $G$ with generators $X$, assume there is a function $V_{G}: \mathcal{D}_{G} \rightarrow \mathbb{R}$ that is smooth on some open domain $\mathcal{D}_{G} \subset \mathbb{R}^{n} \times \cdots \times \mathbb{R}^{n}(|G|$ times $)$ such that 
1) $V_{G}$ may be expressed as the sum of terms corresponding to each component where

$$
\begin{gathered}
V_{g}: \underbrace{\mathbb{R}^{n} \times \cdots \times \mathbb{R}^{n}}_{1+|X| \text { times }} \rightarrow \mathbb{R} \\
V_{G}\left(x_{G}\right)=\sum_{g \in G} V_{g}\left(x_{g}, x_{X g}\right) \\
=\sum_{g \in G} V_{g}\left(x_{g}, w_{s_{1}^{-1} g}^{s_{1}}\left(x_{s_{1}^{-1} g}\right), \ldots, w_{s_{|X|}^{-1} g}^{s_{|X|}}\left(x_{s_{|X|}^{-1} g}\right)\right),
\end{gathered}
$$

for all $x \in \mathcal{D}_{G}$,

2) the individual functions corresponding to each component in $G$ are equal as functions, i.e.,

$$
V_{g_{1}}=V_{g_{2}}=V
$$

for all $g_{1}, g_{2} \in G$, and

3) for any one of the $g \in G$,

$$
\frac{\partial V_{G}}{\partial x_{g}}\left(x_{G}\right)\left(f_{g}\left(x_{g}\right)+\sum_{j=1}^{m} g_{g, j}\left(x_{g}\right) u_{g, j}\left(x_{g}, x_{X g}\right)\right) \leq 0
$$

for all $x_{G} \in \mathcal{D}_{G}$.

Then

1) $\dot{V}_{G}(x) \leq 0$ for all $x \in \mathcal{D}_{G}$ and

2) for any equivalent symmetric system on $\hat{G}$, there is a $V_{\hat{G}}$ such that $\dot{V}_{\hat{G}} \leq 0$ on some open domain, $\mathcal{D}_{\hat{G}}$.

Proof: The full details of the proof are omitted and the reader is referred to [3] for the complete proof. The idea is that $\dot{V}$ is the sum of terms of the form of Equation 14, and the fact that all the terms are negative follows from the symmetry of the system.

This proposition only considers the properties of $\dot{V}$, so we must add the necessary additional conditions to the system to be able to infer stability, which is provided by the following.

Proposition 2: Given a symmetric system on $G$ and a function $V_{G}$ that satisfies the hypotheses of Proposition 1, assume that there exists a positive constant $c$ such that $\Omega_{G}=$ $\left\{x_{G} \in \mathcal{D} \mid V_{G}\left(x_{G}\right) \leq c\right\} \subset \mathcal{D}$ is bounded. Also assume there exists $x_{G} \in \Omega$ such that for the components $\left(x_{g}, x_{X g}, x_{X X g}\right)$ of $x$ corresponding to any one of the $g \in G$

$$
\frac{\partial V_{G}}{\partial x_{g}}\left(x_{G}\right)\left(f_{g}\left(x_{g}\right)+\sum_{j=1}^{m} g_{g, j}\left(x_{g}\right) u_{g, j}\left(x_{g}, x_{X g}\right)\right)=0 .
$$

Then,

1) for the system on $G$, any solution starting in $\Omega_{G}$ approaches the largest invariant set in the set of points in $\Omega_{G}$ where $\dot{V}_{G}=0$ as $t \rightarrow \infty$,

2) for any equivalent system on $\hat{G}$, there exists an $\Omega_{\hat{G}}$ such that as $t \rightarrow \infty$ any solution starting in $\Omega_{\hat{G}}$ approaches the largest invariant set in the set of points in $\Omega_{\hat{G}}$ where $\dot{V}_{\hat{G}}=0$.

Proof: (Sketch) The first result directly follows from Proposition 1 (which ensures $\dot{V} \leq 0$ ) and Lasalle's invariance principle. The second result follows directly from Proposition 1 and Lasalle's invariance principle as long as there exists the set $\Omega_{\hat{G}}$ that is compact that contains some points where $\dot{V}=0$, which naturally follows from the symmetry of the system. The interested reader is referred to [3] for a complete proof.

The following is the main result of the paper.

Proposition 3: Consider a system of the form

$$
\begin{aligned}
\dot{x}_{g}(t)= & f_{g}\left(x_{g}(t)\right) \\
& +\sum_{j=1}^{m_{g}} g_{g, j}\left(x_{g}(t)\right) u_{g, j}\left(x_{g}(t), v_{g}^{s_{1}}(t), \ldots, v_{g}^{s_{|X|}}(t)\right) \\
w_{g}^{s}(t)= & w_{g}^{s}\left(x_{g}(t)\right)
\end{aligned}
$$

which satisfies the requirements of Definition 1 and the hypotheses of Propositions 1 and 2. Assume that

$$
\begin{array}{r}
\frac{\partial V_{G}}{\partial x_{g}}\left(x_{G}\right)\left(f_{g}\left(x_{g}\right)+\sum_{j=1}^{m} g_{g, j}\left(x_{g}\right) u_{g, j}\left(x_{g}, x_{X g}\right)\right) \\
\leq-c_{1} d^{2}\left(x, d V_{0}\right)
\end{array}
$$

for all $x_{G} \in \mathcal{D}_{G}$ where $d\left(x, d V_{0}\right)$ represents the distance from a point $x$ to the set of points where $\dot{V}$ for the symmetric system equals zero and $c_{1}$ is a positive constant.

Given an approximately symmetric system of the form

$$
\begin{aligned}
\dot{x}_{g}(t) & =f_{g}\left(x_{g}(t)\right)+\sum_{j=1}^{m_{g}} g_{g, j}\left(x_{g}(t)\right) u_{g, j}\left(x_{g}(t), x_{X g}(t)\right) \\
& +\hat{f}_{g}\left(x_{g}(t)\right)+\sum_{j=1}^{m} \hat{g}_{g, j}\left(x_{g}(t)\right) u_{g, j}\left(x_{g}(t), x_{X g}(t)\right)
\end{aligned}
$$

$w_{g}^{s}(t)=w_{g}^{s}\left(x_{g}(t)\right)$

if $d\left(x, d V_{0}\right)$ represents the distance from a point $x$ to the set of points where $\dot{V}$ for the symmetric system equals zero, and if there exist positive constants such that

$$
\begin{aligned}
\left\|\frac{\partial V}{\partial x_{g}}\left(x_{G}\right)\right\| & \leq c_{2} d\left(x, d V_{0}\right), \\
\left\|\hat{f}_{g}\left(x_{g}\right)+\hat{g}_{g}\left(x_{g}\right) u\left(x_{g}, x_{X g}\right)\right\| & \leq c_{3} d\left(x, d V_{0}\right)
\end{aligned}
$$

for all $x_{G} \in \mathcal{D}_{G}$ such that $c_{2} c_{3} / c_{1}<1$ then any solution starting in $\Omega_{G}$ approaches the largest invariant set in the set of points where $\dot{V}_{G}=0$ as $t \rightarrow \infty$. Correspondingly for any equivalent symmetric system, any solution starting in $\Omega_{\hat{G}}$ approaches the largest invariant set in the set of points where $\dot{V}_{\hat{G}}=0$ as $t \rightarrow \infty$.

Proof: The only thing needed in order to utilize Proposition 1, is to ensure that Equation 14 is satisfied. For 
the approximately symmetric system

$$
\begin{aligned}
& \frac{\partial V_{G}}{\partial x_{g}}\left(x_{G}\right) \cdot\left(f_{g}\left(x_{g}(t)\right)+\sum_{j=1}^{m_{g}} g_{g, j}\left(x_{g}(t)\right) u_{g, j}\left(x_{g}(t), x_{X g}\right)\right. \\
& \left.\quad+\hat{f}_{g}\left(x_{g}(t)\right)+\sum_{j=1}^{m} \hat{g}_{g, j}\left(x_{g}(t)\right) u_{g, j}\left(x_{g}(t), x_{X g}(t)\right)\right) \\
& \quad \leq-c_{1} d^{2}\left(x, d V_{0}\right)+c_{2} c_{3} d^{2}\left(x, d V_{0}\right) \\
& \quad \leq\left(c_{2} c_{3}-c_{1}\right) d^{2}\left(x, d V_{0}\right) \\
& \quad \leq 0
\end{aligned}
$$

for all $x_{G} \in \mathcal{D}_{G}$. Thus all the requirements of Propositions 1 and 2 are satisfied.

It may be the case that differences between an approximately symmetric system and an exactly symmetric system that are even more structured. The following corollary considers such a case. that

Corollary 1: If there exists a positive constant, $c_{2}$ such

$$
\begin{aligned}
& \| \frac{\partial V_{G}}{\partial x_{g}}\left(x_{G}\right) \cdot\left(\hat{f}_{g}\left(x_{g}(t)\right)\right. \\
& \left.+\sum_{j=1}^{m} \hat{g}_{g, j}\left(x_{g}(t)\right) u_{g, j}\left(x_{g}(t), x_{X g}(t)\right)\right) \| \leq c_{2} d^{2}\left(x_{g}, d V_{0}\right)
\end{aligned}
$$

for all $x_{G} \in \mathcal{D}_{G}$ such that $c_{2}<c_{1}$, and all the conditions of Proposition 3 are satisfied other than Equations 18 and 19, then then any solution starting in $\Omega_{G}$ approaches the largest invariant set in the set of points where $\dot{V}_{G}=0$ as $t \rightarrow \infty$.

Proof: Similar to the proof to Proposition 3

$$
\begin{aligned}
& \frac{\partial V_{G}}{\partial x_{g}}\left(x_{G}\right) \cdot\left(f_{g}\left(x_{g}(t)\right)+\sum_{j=1}^{m_{g}} g_{g, j}\left(x_{g}(t)\right) u_{g, j}\left(x_{g}(t), x_{X g}\right)\right. \\
& \left.\quad+\hat{f}_{g}\left(x_{g}(t)\right)+\sum_{j=1}^{m} \hat{g}_{g, j}\left(x_{g}(t)\right) u_{g, j}\left(x_{g}(t), x_{X g}(t)\right)\right) \\
& \quad \leq-c_{1} d^{2}\left(x, d V_{0}\right)+c_{2} d^{2}\left(x, d V_{0}\right) \\
& \quad \leq\left(c_{2}-c_{1}\right) d^{2}\left(x, d V_{0}\right) \\
& \quad \leq 0
\end{aligned}
$$

Thus, all the requirements of Propositions 1 and 2 are satisfied.

\section{EXAMPLE}

This section will complete Example 2.

Example 4: Continuing Example 2, for a fleet of 5 agents, note that

$$
X=\{-2,-1,0,1,2\}
$$

is a group with the group operation of addition and the relation $s^{5}=0$. Define the Lyapunov function on $G=X$ as

$$
\begin{aligned}
V_{G}\left(x_{G}\right)= & \sum_{i=1}^{5} V_{i}\left(x_{i}, x_{i-2}, x_{i-1}, x_{i+1}, x_{i+2}\right) \\
= & \sum_{i=1}^{5} \frac{1}{2}\left[\left(\dot{x}_{i}^{2}+\dot{y}_{i}^{2}\right)+k_{o}\left(\sqrt{x_{i}^{2}+y_{i}^{2}}-r_{i}\right)^{2}\right. \\
& \left.+\sum_{j}\left(\sqrt{\left(x_{i}-x_{j}\right)^{2}+\left(y_{i}-y_{j}\right)^{2}}-d_{i j}\right)^{2}\right]
\end{aligned}
$$

where $j \in\{i-2, i-1, i+1, i+2\}, d_{i j}$ is the desired distance between robots and $r_{i}$ is the desired distance of robot $i$ from the origin, as defined previously. Note that $V_{G}$ is smooth everywhere, by construction, $V_{G}$ is the sum of individual terms of the form $V_{i}\left(x_{i}, x_{i-2}, x_{i-1}, x_{i+1}, x_{i+2}\right)$, and by construction, $V_{i}=V_{j}$ as functions.

Next we show that Equation 14 is satisfied. A detailed computation for $\frac{\partial V_{G}}{\partial x_{i}}\left(f_{i}+\sum_{j} g_{i, j} u_{i, j}\right)$ gives

$$
\frac{\partial V_{G}}{\partial x_{i}} \cdot\left(f_{i}+\sum_{j} g_{i, j} u_{i, j}\right)=-k_{d}\left(\dot{x}_{i}^{2}+\dot{y}_{i}^{2}\right),
$$

which is clearly negative semidefinite. Hence, by Proposition $1, \dot{V}_{G}$ is negative semidefinite as is $\dot{V}_{\hat{G}}$ for any equivalent system.

Now, we show that the hypotheses of Proposition 2 are met. Because of the first two terms in $V_{i}$, each $V_{i}$ is radially unbounded. Hence, for any finite initial conditions, there exists a constant, $c$, such that the initial conditions are in the set $\Omega_{G}$ as defined in Proposition 2. Any state with all robots at rest are such that $\dot{V}_{G}=0$. Finally, Equation 14 is satisfied everywhere. Hence, by Proposition 2, we can conclude that the system approaches the largest invariant set such that $\dot{V}=0$, which is the set that contains the desired formation. The same is true for any equivalent system.

Simulation results for a five-agent system are illustrated in Figures 3 and 4 with $k_{d}=0.5$ and $k_{o}=0.01$. Figure 3 shows the trajectories for the individual agents, and Figure 4 shows the final configuration. In all the simulations in this paper, the $\times$ symbol indicates an initial condition, a $\circ$ symbol represents a final configuration and the trajectories over time are indicated by the lines.

Now, to illustrate the application of the main result in this paper, we break the symmetry by adding to each robot a destabilizing negative damping force that is different for each robot. Specifically, for the $i$ th robot take the equation of motion to be

$$
\frac{d}{d t}\left[\begin{array}{c}
x_{i} \\
\dot{x}_{i} \\
y_{i} \\
\dot{y}_{i}
\end{array}\right]=\left[\begin{array}{c}
\dot{x}_{i} \\
0 \\
\dot{y}_{i} \\
0
\end{array}\right]+\left[\begin{array}{c}
0 \\
k_{i} \dot{x}_{i} \\
0 \\
k_{i} \dot{y}_{i}
\end{array}\right]+\left[\begin{array}{l}
0 \\
1 \\
0 \\
0
\end{array}\right] u_{i, 1}+\left[\begin{array}{l}
0 \\
0 \\
0 \\
1
\end{array}\right] u_{i, 2}
$$

where the second term on the right-hand side is $\hat{f}\left(x_{g}\right)$. Referring to Equation 22, it is clear that Equation 16 is satisfied because the set of points where $\dot{V}=0$ are where 


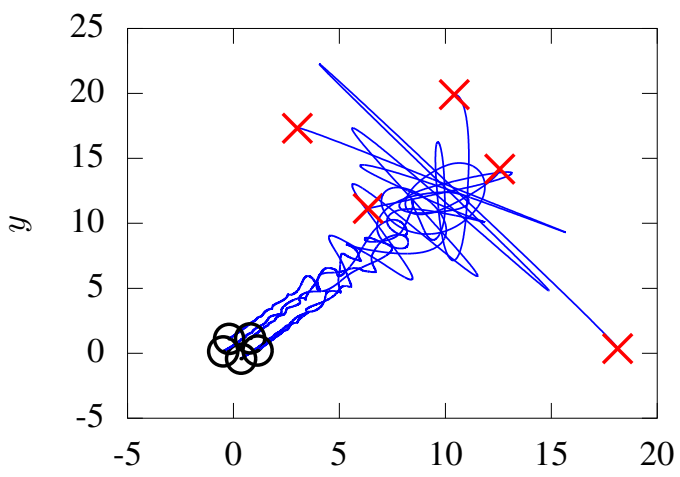

Fig. 3. Trajectories for distributed control for a five-vehicle system.

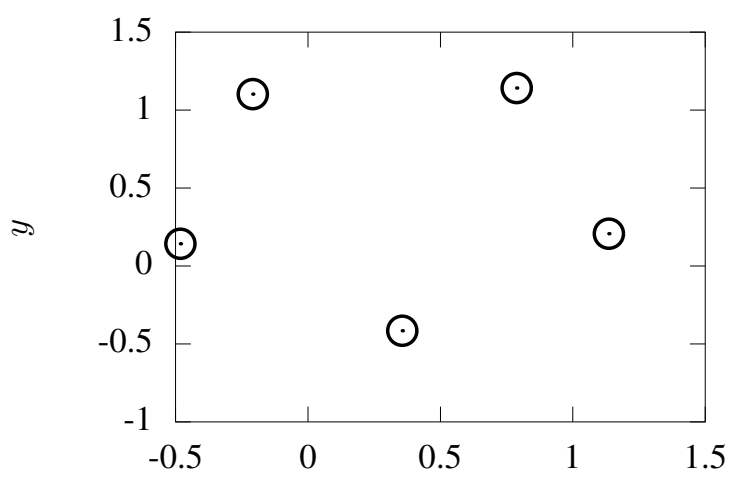

Fig. 4. Final formation ${ }^{x}$ for distributed control for a five-vehicle system.

the velocity of each robot is zero. Hence, the distance from this set is given by

$$
d\left(x_{i}, x_{X i}\right)=\sqrt{\dot{x}_{i}^{2}+\dot{y}_{i}^{2}} .
$$

Hence, $c_{1}=k_{d}$. Furthermore,

$$
\frac{\partial V_{g}}{\partial x_{i}} \cdot \hat{f}\left(x_{i}\right)=k_{i}\left(\dot{x}_{i}^{2}+\dot{y}_{i}^{2}\right)
$$

so we may use Corollary 1 with $c_{2}=k_{i}$. Hence, as long as each $k_{i} \leq k_{d}$, the conditions of Corollary 1 are satisfied and all solutions should converge to the desired formation. This is illustrated for a five robot system in Figures 5 (trajectories) and 6 (final positions) where $k_{i}=\left[\begin{array}{lllll}.2 & .4 & .1 & .25 & .45\end{array}\right]$. Furthermore any other larger system with nine robots that is equivalent to this system will also converge to the desired formation. This is illustrated in Figures 7 and 8 with $k_{i}=$ $\left[\begin{array}{llllllll}.2 & .4 & .1 & .25 & .45 & .1 & .1 & .2\end{array}\right]$.

\section{CONClusions And Future Work}

This paper extends the prior work of the author which considers stability of classes of symmetric systems. The value of that prior work was that if a controls engineer checks the stability of only one symmetric system, then it is automatically the case that stability of all symmetric systems

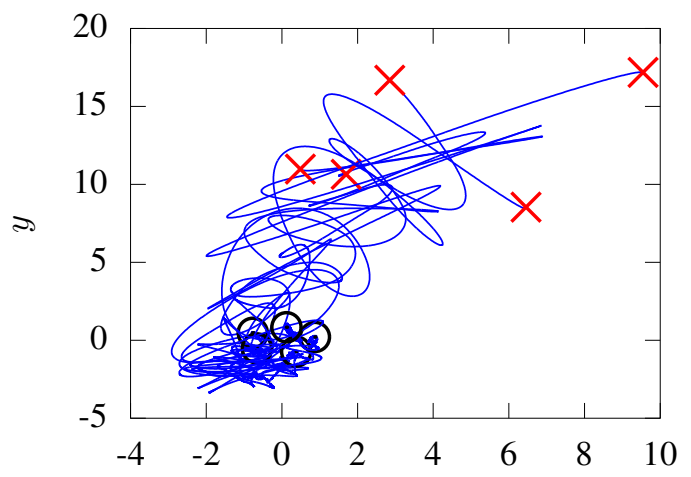

Fig. 5. Trajectories for distributed control for an approximately symmetric five-vehicle system.

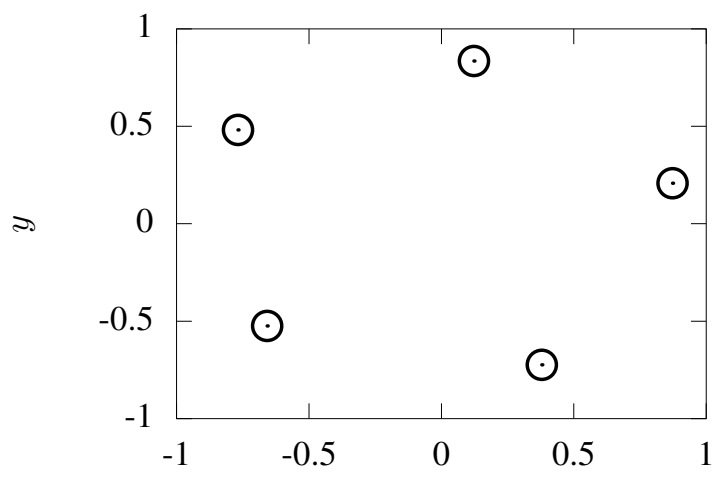

Fig. 6. Final formation for distributed control for an approximately symmetric five-vehicle system.

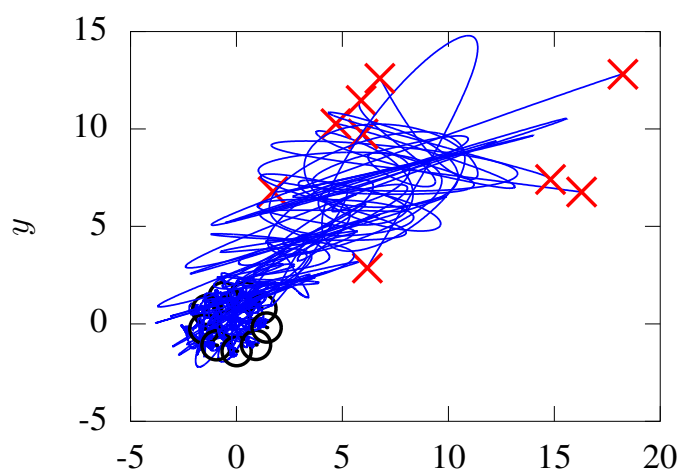

Fig. 7. Trajectories for $\stackrel{x}{d i s t r i b u t e d ~ c o n t r o l ~ f o r ~ a n ~}$ approximately symmetric nine-vehicle system. 


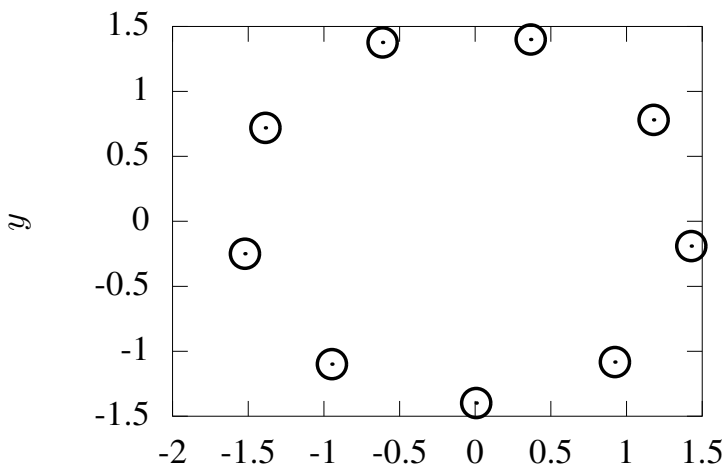

Fig. 8. Final formation ${ }^{x}$ for distributed control for an approximately symmetric nine-vehicle system.

equivalent to it is guaranteed. This paper extends those results to approximately symmetric systems. In this paper, conditions on the difference between exactly symmetric systems and approximately symmetric systems are determined are presented, which, if satisfied, guarantee stability for a class of approximately symmetric systems.

Future work is directed toward extending these results in a variety of ways. First, the result in this paper still ensures stability. A natural extension is when the system is no longer stable, but rather bounded, and our focus in that case is on conditions relating $\dot{V}$ and bounds on the symmetry-breaking terms. Also, another class of problems we are considering are when the symmetry-breaking arises from persistent, nonautonomous inputs, which would arise in applications such as surveillance where agents are to maintain a nominally equal spread over a defined area, but where individual agents need to maintain a pattern of motion to ensure coverage of their assigned areas.

\section{REFERENCES}

[1] Janos Sztipanovits, Xenofon Koutsoukos, Gabor Karsai, Nicholas Kottenstette, Panos Antsaklis, Bill Goodwine Vijay Gupta, John Baras, and Shige Wang. Toward a science of cyber-physical system integration. Proceedings of the IEEE, 2011.

[2] Jacques Julliand, Hassan Mountassir, and Emilie Oudot. Composability, compatibility, compositionality: automatic preservation of timed properties during incremental development. Technical report, UFR Sciences et Techniques, 2007.

[3] Bill Goodwine and Panos Antsaklis. Multiagent compositional stability exploiting system symmetries. Provisionally accepted for publication in Automatica. Preprint available: http://controls. ame.nd. edu/ bill/sym.pdf.

[4] M. Brett McMickell and Bill Goodwine. Reduction and nonlinear controllability of symmetric distributed systems. International Journal of Control, 76(18):1809-1822, 2003.

[5] M. Brett McMickell and Bill Goodwine. Reduction and controllability of robotic systems with drift. In Proceedings of the 2002 IEEE International Conference on Robotics and Automation, May 2002.

[6] M. Brett McMickell and Bill Goodwine. Motion planning for symmetric distributed robotic systems. In 2003 IEEE International Conference on Robotics and Automation, September 2003.

[7] M. Brett McMickell and Bill Goodwine. Motion planning for nonlinear symmetric distributeed robotic systems. International Journal of Robotics Research, 26(10):1025-1041, October 2007.

[8] M. Brett McMickell, Bill Goodwine, and Luis Antonio Montestruque. Micabot: A robotic platform for large-scale distributed robotics. In 2003 IEEE International Conference on Robotics and Automation, September 2003.

[9] M. Brett McMickell and Bill Goodwine. Reductionand controllability of symmetric distributed systems with robotic applications. In International Conference on Intelligent Robotics and Systems, volume 3, pages 1232-1236. IEEE/RSJ, October 2001.

[10] Richard M. Murray. Recent research in cooperative control of multivehicle systems. Journal of Dynamical Systems, Measurement and Control, 129:571-583, 2007.

[11] Ali Jadbabaie, Jie Lin, and A. Stephen Morse. Coordination of groups of mobile autonomous agents using nearest neighbor rules. IEEE Transactions on Automatic Control, 48(6):988-1001, 2003.

[12] Wei Ren, Randal W. Beard, and Ella M. Atkins. Information consensus in multivehicle cooperative control. IEEE Control Systems Magazine, pages 71-82, April 2007.

[13] J. Alexander Fax and Richard M. Murray. Information flow and cooperative control of vehicle formations. IEEE Transactions on Automatic Control, 49(9):1465- 1476, 2004.

[14] Elon Rimon and Daniel E. Koditschek. Exact robot navigation using artificial potential functions. IEEE Transactions on Robotics and Automation, 8(5):501-518, 1992.

[15] N.E. Leonard and E. Fiorelli. Virtual leaders, artificial potentials, and coordinated cont. pages 2968-2973, December 2001.

[16] Reza Olfati-Saber and Richard M. Murray. Distributed cooperative control of multiple vehicle formations using structural potential functions. In Proceedings of the 2002 IFAC World Congress, July 2002.

[17] Petter Ögren, Magnus Egerstedt, and Xiaoming Hu. A control lyapunov function approach to multiagent coordination. IEEE Transactions on Robotics and Automation, 18(5):847-851, October 2002.

[18] Randy Cogill, Sanjay Lall, and Pablo A. Parrilo. Structured semidefinite programs for the control of symmetric systems. Automatica, 44:1411-1417, 2008

[19] A.J. van der Schaft. Symmetries in optimal control. SIAM Journal of Control and Optimization, 25(2):245-259, 1987.

[20] Srihari Govindan, Arndt von Schemde, and Bernhard von Stengel. Symmetry and $p$-stability. International Journal of Game Theory, 32:359-369, 2003.

[21] Raffaello D'Andrea and Geir E. Dullerud. Distributed control design for spatially interconnected systems. IEEE Transactions on Automatic Control, 48(9):1478-1495, September 2003.

[22] Joseph J. Rotman. An Introduction to the Theory of Groups. SpringerVerlag, fourth edition, 1995 